\title{
POSITIVE SOLUTIONS FOR BOUNDARY VALUE PROBLEM OF FRACTIONAL DIFFERENTIAL EQUATION IN BANACH SPACES
}

\author{
XUPING ZHANG ${ }^{1}$, YOUHUI SU², AND YIBO KONG ${ }^{3}$ \\ ${ }^{1}$ Department of Mathematics, Northwest Normal University, Lanzhou \\ 730070, P.R. China \\ ${ }^{2}$ School of Mathematics and Statistics, Xuzhou University of Technology, \\ Xuzhou 221018, P.R. China \\ ${ }^{3}$ School of Finance and business, Lanzhou Resources and Environment \\ Vic-Tech College, Lanzhou 730030, P.R. China
}

\begin{abstract}
In this paper, we consider the existence of positive solutions for the following boundary value problem of fractional differential equations in Banach space $E$

$$
\left\{\begin{array}{c}
D^{\alpha} u(t)=f(t, u(t)), \quad t \in I, \\
\lim _{t \rightarrow 0^{+}} t^{1-\alpha} u(t)=\omega^{1-\alpha} u(\omega),
\end{array}\right.
$$

where $0<\alpha \leq 1$ is real number, $I=(0, \omega], D^{\alpha}$ is the Riemann-Liouville fractional derivative, $f$ : $I \times K \rightarrow K$ is continuous, $K$ is a normal cone in Banach space $E$. Under more general growth and noncompactness measure conditions about nonlinearity $f$, we obtained the existence of positive solutions by applying the fixed point index theory of condensing mapping.
\end{abstract}

AMS (MOS) Subject Classification. 34B15, 34B18, 47H08.

Key Words and Phrases. Fractional differential equation, Positive solutions, Cone, Measure of noncompactness, Condensing mapping.

\section{INTRODUCTION}

Fractional differential equations derive in various field such as physics, chemistry and engineering. And it has been of great interest for the last decade (see[1]-[4]). Boundary value problem of fractional differential equations have been investigated in many paper (see [5]-[14] and the reference therein). In view of the fact that only positive solutions are meaningful in practical problem, we discuss the existence of positive for boundary value problems of fractional differential equations in this paper.

Using fixed point theory on cone, Bai [15] discuss the existence results of positive solutions for the following boundary value problem of fractional differential equation in

Received January 1, 2021

1056-2176 \$15.00 (C)Dynamic Publishers, Inc.

www.dynamicpublishers.org;

www.dynamicpublishers.com;

https://doi.org/10.46719/dsa202130.09.04. 
real spaces

$$
\left\{\begin{array}{l}
D_{0^{+}}^{\alpha} u(t)+f(t, u(t))=0, \quad 0<t<1, \\
u(0)=u(1)=0
\end{array}\right.
$$

where $1<\alpha \leq 2$ is real numbers, $D_{0^{+}}^{\alpha}$ is the standard Riemann-Liouville fractional derivative, the function $f$ is continuous on $[0,1] \times[0,+\infty)$.

In [16], the existence of positive solutions for the nonlinear fractional differential equations with Dirichlet problem

$$
\left\{\begin{array}{l}
D^{\alpha} u(t)-\lambda u(t)+f\left(t, t^{1-\alpha} u(t)\right)=0, \quad 0<t<1, \\
\lim _{t \rightarrow 0^{+}} t^{2-\alpha} u(t)=u(1)=0
\end{array}\right.
$$

is investigated by constructing a proper cone, where $1<\alpha \leq 2, D^{\alpha}$ is the standard Riemann-Liouville fractional derivative, the function $f$ is continuous on $[0,1] \times[0,+\infty)$.

However, the above-mentioned results are discussed in real spaces. Because of the essential differential between infinite dimensional space and finite dimensional space, the existence of solutions of ordinary differential equations is no longer valid in abstract Banach space. We should mention that $\mathrm{Li}$ and Guo [17] discussed the existence of positive solutions for two-point boundary value problem of second order nonlinear differential equations in Banach spaces by using the fixed point index theory on cones. The authors replace the limit condition with the order condition which is easy to satisfy, describes the growth condition of the nonlinear term, and improves the conclusion of reference [18]. Motivated by the aforementioned works, in this article, under more general conditions of growth and noncompactness measure about the nonlinear term $f$, combining with fixed point index theory of condensing mapping in cone, we study the existence of positive solutions for the following boundary value problem of fractional differential equation in Banach spaces $E$

$$
\left\{\begin{array}{l}
D^{\alpha} u(t)=f(t, u(t)), \quad t \in I, \\
\lim _{t \rightarrow 0^{+}} t^{1-\alpha} u(t)=\omega^{1-\alpha} u(\omega),
\end{array}\right.
$$

where $0<\alpha \leq 1$ is a real number, $I=(0, \omega], D^{\alpha}$ is the Riemann-Liouville fractional derivative, and $f:[0, \omega] \times K \rightarrow K$ is a continuous, $K$ is a normal come in Banach space E.

\section{Preliminaries}

Let $E$ be a Banach space, $I=[0, \omega]$, we use $C(I, E)$ to denote the Banach space of all continuous function on interval $I$ with the norm $\|u\|_{C}=\max _{t \in I}\|u(t)\|$. In our further consideration we utilize its generalization, for $1-\alpha>0, u \in C(I, E)$, denote

$$
\left.t^{1-\alpha} u(t)\right|_{t=0}=\lim _{t \rightarrow 0^{+}} t^{1-\alpha} u(t), \quad C_{1-\alpha}(I, E)=\left\{u \in C(I, E) \mid t^{1-\alpha} u(t) \in C(I, E)\right\} .
$$


Then $C_{1-\alpha}(I, E)$ equipped with the norm $\|u\|_{C_{1-\alpha}}=\left\|t^{1-\alpha} u(t)\right\|_{C}$. It is easy to verify that $C_{1-\alpha}(I, E)$ is a Banach space. Evidently, $C_{1-\alpha}(I, E)$ is an ordered Banach space induced by the convex cone $C_{1-\alpha}(I, K)=\left\{u \in C_{1-\alpha}(I, E) \mid u(t) \in K\right\}$, and $C_{1-\alpha}(I, K)$ is also a normal cone in $C_{1-\alpha}(I, E)$.

In this article, we denote by $\mu(\cdot), \mu_{C}(\cdot)$ and $\mu_{C_{1-\alpha}}(\cdot)$ the Kuratowski measure of noncompactness on the bounded set of $E, C(I, E)$ and $C_{1-\alpha}(I, E)$, respectively. For any $B \subset C(I, E)$ and $t \in I$, set $B(t)=\{u(t) \mid u \in B\} \subset E$. If $B$ is bounded in $C(I, E)$, then $B(t)$ is bounded in $E$, and $\mu(B(t)) \leq \mu_{C}(B)$. For the details of the properties of the measure of noncompactness, we refer to the monographs $[19,20]$.

For the convenience of the reader, first, we present the necessary lemmas.

The Mittag-Leffler function plays a similar role in fractional calculus as the exponential function in the theory of integer-order differential equation. Thus, the Mittag-Leffler function in two parameters is defined as [21]

$$
E_{\alpha, \beta}(z)=\sum_{k=0}^{\infty} \frac{z^{k}}{\Gamma(\alpha k+\beta)} \quad z \in \mathbb{R}, \quad \alpha, \beta>0 .
$$

Note that the series converges uniformly in $\mathbb{R}$.

Lemma 2.1. ([22]) Let $0<\alpha \leq 1, \beta, \gamma>0, M \in \mathbb{R}$ and $a \in \mathbb{R}$. Then it holds

(i) $E_{\alpha, 2 \alpha}\left(M t^{\alpha}\right)=M^{-1} t^{-\alpha}\left(E_{\alpha, \alpha}\left(M t^{\alpha}\right)-1 / \Gamma(\alpha)\right)$;

(ii) $I_{a}^{\gamma}(t-a)^{\beta-1} E_{\alpha, \beta}\left(M(t-a)^{\alpha}\right)=(t-a)^{\beta+\gamma-1} E_{\alpha, \beta+\gamma}\left(M(t-a)^{\alpha}\right)$ for $t>a$;

(iii) $E_{\alpha, \alpha}\left(M t^{\alpha}\right)$ is decreasing in $t$ for $M<0$ and increasing for $M>0$ for all $t>0$.

Lemma 2.2. ([23]) Let $B=\left\{u_{n}\right\} \subset C_{1-\alpha}(I, E)$ be a bounded and countable set, then $\alpha(B(t))$ is Lebesgue integral on $I$, and

$$
\mu\left(\left\{\int_{I} u_{n}(t) d t \mid n \in \mathbb{N}\right\}\right) \leq 2 \int_{I} \mu(B(t)) d t
$$

Lemma 2.3. ([24], [25]) Let $D \subset E$ be bounded. Then exist a countable set $D_{0} \subset D$, such that $\mu(D)<2 \mu\left(D_{0}\right)$.

Lemma 2.4. ([26]) Let $B \subset C_{1-\alpha}(I, E)$ be bounded and equicontinuous. Then $\mu(B(t))$ is continuous on $I$, and

$$
\mu_{C_{1-\alpha}}(B)=\max _{t \in I} \mu(B(t))=\mu_{C_{1-\alpha}}(B(I)) .
$$

Obviously, the boundary value problem (1) is equivalent to the following

$$
\left\{\begin{array}{l}
D^{\alpha} u(t)+M u(t)=f(t, u(t))+M u(t), \quad t \in I, \\
\lim _{t \rightarrow 0^{+}} t^{1-\alpha} u(t)=\omega^{1-\alpha} u(\omega),
\end{array}\right.
$$

where $M>0$ is real number.

To prove our main results, we consider the boundary value problem (2) the corresponding linear problem, and give the following Lemma. 
Lemma 2.5. For any $h \in C_{1-\alpha}(I, E)$, the linear boundary value problem of fractional differential equation in Banach spaces

$$
\left\{\begin{array}{l}
D^{\alpha} u(t)+M u(t)=h(t), \quad t \in I \\
\lim _{t \rightarrow 0^{+}} t^{1-\alpha} u(t)=\omega^{1-\alpha} u(\omega)
\end{array}\right.
$$

has a unique solution

$$
u(t)=\int_{0}^{\omega} G(t, s) h(s) d s:=S h(t)
$$

where

$$
G(t, s)= \begin{cases}\frac{\omega^{1-\alpha} \Gamma(\alpha) E_{\alpha, \alpha}\left(-M t^{\alpha}\right) E_{\alpha, \alpha}\left(-M(\omega-s)^{\alpha}\right)}{\left(1-\Gamma(\alpha) E_{\alpha, \alpha}\left(-M \omega^{\alpha}\right)\right)(\omega-s)^{1-\alpha} t^{1-\alpha}}+\frac{E_{\alpha, \alpha}\left(-M(t-s)^{\alpha}\right)}{(t-s)^{1-\alpha}}, & 0 \leq s<t \leq \omega \\ \frac{\omega^{1-\alpha} \Gamma(\alpha) E_{\alpha, \alpha}\left(-M t^{\alpha}\right) E_{\alpha, \alpha}\left(-M(\omega-s)^{\alpha}\right)}{\left(1-\Gamma(\alpha) E_{\alpha, \alpha}\left(-M \omega^{\alpha}\right)\right)(\omega-s)^{1-\alpha} t^{1-\alpha}}, & 0<t \leq s<\omega\end{cases}
$$

Proof. We can verify directly that the function $u \in C_{1-\alpha}(I, E)$ defined by the expression (4) is a solution of the linear boundary value problem (3). Next, we prove that $u$ is uniqueness of solution. Assume that $u_{1}, u_{2} \in C_{1-\alpha}(I, E)$ are two solutions of the linear boundary value problem (3). for any $\varphi \in E^{*}$ (The dual space of $E^{*}$ is $E$ ), note $r(t)=\varphi\left(u_{1}(t)-u_{2}(t)\right)$, by means of the reference [7], then the scalar linear boundary value problem

$$
\left\{\begin{array}{l}
D^{\alpha} r(t)+M r(t)=0, \quad t \in I \\
\lim _{t \rightarrow 0^{+}} t^{1-\alpha} r(t)=\omega^{1-\alpha} r(\omega)
\end{array}\right.
$$

has only zero solution. Since $r(t) \equiv 0$, without loss of generality of $\varphi \in E^{*}$, we get $u_{1}(t)-u_{2}(t)=0$, namely, $u_{1}(t)=u_{2}(t)$ in $I$. Hence, the linear boundary value problem (3) has a unique solution $u(t)$.

Conversely, we can verify directly that the function $u \in C_{1-\alpha}(I, E)$ defined by (4) is a solution of the linear problem (3). Therefore, the conclusion of Lemma 2.5 holds.

Lemma 2.6. Let $0<\alpha \leq 1$ and $M>0$, then the Green's function (5) satisfies

$$
t^{1-\alpha} \int_{0}^{\omega} G(t, s) s^{\alpha-1} d s=\frac{1}{M}, \quad t, s \in[0, \omega]
$$


Proof. Employing the results of Lemma 2.1, we have

$$
\begin{aligned}
t^{1-\alpha} \int_{0}^{\omega} G(t, s) s^{\alpha-1} d s= & \int_{0}^{\omega} \frac{\omega^{1-\alpha} \Gamma(\alpha) E_{\alpha, \alpha}\left(-M t^{\alpha}\right) E_{\alpha, \alpha}\left(-M(\omega-s)^{\alpha}\right)}{\left(1-\Gamma(\alpha) E_{\alpha, \alpha}\left(-M \omega^{\alpha}\right)\right)(\omega-s)^{1-\alpha}} s^{\alpha-1} d s \\
& +t^{1-\alpha} \int_{0}^{t} \frac{E_{\alpha, \alpha}\left(-M(t-s)^{\alpha}\right)}{(t-s)^{1-\alpha}} s^{\alpha-1} d s \\
= & \frac{\omega^{1-\alpha} \Gamma(\alpha) E_{\alpha, \alpha}\left(-M t^{\alpha}\right)}{1-\Gamma(\alpha) E_{\alpha, \alpha}\left(-M \omega^{\alpha}\right)} \int_{0}^{\omega} \frac{E_{\alpha, \alpha}\left(-M(\omega-s)^{\alpha}\right)}{(\omega-s)^{1-\alpha}} s^{\alpha-1} d s \\
& +t^{1-\alpha} \int_{0}^{t} \frac{E_{\alpha, \alpha}\left(-M(t-s)^{\alpha}\right)}{(t-s)^{1-\alpha}} s^{\alpha-1} d s \\
= & \left.\frac{\omega^{1-\alpha} \Gamma(\alpha)^{2} E_{\alpha, \alpha}\left(-M t^{\alpha}\right)}{1-\Gamma(\alpha) E_{\alpha, \alpha}\left(-M \omega^{\alpha}\right)}\left(I_{0}^{\alpha} t^{\alpha-1} E_{\alpha, \alpha}\left(-M t^{\alpha}\right)\right)\right|_{t=\omega} \\
& +\Gamma(\alpha) t^{1-\alpha}\left(I_{0}^{\alpha} t^{\alpha-1} E_{\alpha, \alpha}\left(-M t^{\alpha}\right)\right) \\
= & \frac{-\Gamma(\alpha)^{2} E_{\alpha, \alpha}\left(-M t^{\alpha}\right)}{M\left(1-\Gamma(\alpha) E_{\alpha, \alpha}\left(-M \omega^{\alpha}\right)\right)}\left(E_{\alpha, \alpha}\left(-M \omega^{\alpha}\right)-\frac{1}{\Gamma(\alpha)}\right) \\
& -\frac{\Gamma(\alpha)\left(E_{\alpha, \alpha}\left(-M t^{\alpha}\right)-\frac{1}{\Gamma(\alpha)}\right)}{M}=\frac{1}{M} .
\end{aligned}
$$

This completes the proof.

Lemma 2.7. For any $h \in C_{1-\alpha}(I, E)$, then the norm of solution operator $S$ satisfies

$$
\|S\|_{C_{1-\alpha}} \leq \frac{1}{M}
$$

Proof. For any $h \in C_{1-\alpha}(I, E)$, due to the definition of operator $S$ and the Lemma 2.6, we get that

$$
\begin{aligned}
\left\|t^{1-\alpha} S h(t)\right\| & =\left\|t^{1-\alpha} \int_{0}^{\omega} G(t, s) h(s) d s\right\|=\left\|t^{1-\alpha} \int_{0}^{\omega} G(t, s) s^{\alpha-1} s^{1-\alpha} h(s) d s\right\| \\
& \leq t^{1-\alpha} \int_{0}^{\omega} G(t, s) s^{\alpha-1}\left\|s^{1-\alpha} h(s)\right\| d s \\
& \leq t^{1-\alpha} \int_{0}^{\omega} G(t, s) s^{\alpha-1} d s\|h\|_{C_{1-\alpha}}=\frac{1}{M}\|h\|_{C_{1-\alpha}},
\end{aligned}
$$

which means that $\|S h\|_{C_{1-\alpha}} \leq \frac{1}{M}\|h\|_{C_{1-\alpha}}$. Hence, (6) holds.

Now, let us prove two additional inequalities for Green's function $G(t, s)$.

Lemma 2.8. Let $G(t, s)$ be Green's function related to problem (3), given by expression (5). Then there exist a positive constant $M$ and a function $m(t) \in C_{1-\alpha}[0, \omega]$ such that $m(t) \geq 0$ for all $t \in(0, \omega)$, for which the following inequalities hold

$$
m(t) \leq \frac{t^{1-\alpha} G(t, s)}{e_{\alpha}^{-M(\omega-s)}} \leq M, \quad t, s \in(0, \omega)
$$


where $e_{\alpha}^{-M(\omega-s)}=(\omega-s)^{\alpha-1} E_{\alpha, \alpha}\left(-M(\omega-s)^{\alpha}\right)$.

Proof. Denote $h(t, s)=\frac{t^{1-\alpha} G(t, s)}{e_{\alpha}^{-M(\omega-s)}}$, then $h(t, s)$ is continuous on $[0, \omega] \times[0, \omega)$. Moreover, for all $t \in[0, \omega]$ the limits

$$
\begin{aligned}
l_{1}(t) & =\lim _{s \rightarrow \omega} h(t, s)=\lim _{s \rightarrow \omega} \frac{t^{1-\alpha} G(t, s)}{e_{\alpha}^{-M(\omega-s)}} \\
& =\lim _{s \rightarrow \omega} \frac{t^{1-\alpha} \Gamma(\alpha) \omega^{1-\alpha} E_{\alpha, \alpha}\left(-M t^{\alpha}\right) E_{\alpha, \alpha}\left(-M(\omega-s)^{\alpha}\right)}{\left(1-\Gamma(\alpha) E_{\alpha, \alpha}\left(-M \omega^{\alpha}\right)\right)(\omega-s)^{1-\alpha} t^{1-\alpha} e_{\alpha}^{-M(\omega-s)}} \\
& =\lim _{s \rightarrow \omega} \frac{\Gamma(\alpha) \omega^{1-\alpha} E_{\alpha, \alpha}\left(-M t^{\alpha}\right) e_{\alpha}^{-M(\omega-s)}}{\left(1-\Gamma(\alpha) E_{\alpha, \alpha}\left(-M \omega^{\alpha}\right)\right) e_{\alpha}^{-M(\omega-s)}}=\frac{\omega^{1-\alpha} \Gamma(\alpha) E_{\alpha, \alpha}\left(-M t^{\alpha}\right)}{1-\Gamma(\alpha) E_{\alpha, \alpha}\left(-M \omega^{\alpha}\right)}
\end{aligned}
$$

exist and are finite, so $h(t, s)$ has removable discontinuities at $s=\omega$, and we can extend it continuously to a function $\widetilde{h}(t, s)$ on $[0, \omega] \times[0, \omega]$.

Therefore, $m(t)=\min _{s \in[0, \omega]}\{\widetilde{h}(t, s)\}$ is a continuous function such that $0 \leq m(t) \leq$ $\widetilde{h}(t, s) \leq M$ for all $t, s \in[0, \omega]$, where $M=\max _{(t, s) \in[0, \omega] \times[0, \omega]} \widetilde{h}(t, s)$. That is, the inequality holds.

Lemma 2.9. Let $h \in C_{1-\alpha}(I, K)$, then boundary value problem of fractional differential equation (1) the solution of the corresponding linear equation (3) is $u(t)=S h(t)$, the following inequalities are satisfied

$$
t^{1-\alpha} u(t) \geq \frac{m(t)}{M} \eta^{1-\alpha} u(\eta), \quad t, \eta \in I .
$$

Proof. From Lemma 2.8 and (4), we have

$$
\begin{aligned}
t^{1-\alpha} u(t) & =t^{1-\alpha} \int_{0}^{\omega} G(t, s) h(s) d s \geq m(t) \int_{0}^{\omega} e_{\alpha}^{-M(\omega-s)} h(s) d s \\
& \geq \frac{m(t)}{M} \int_{0}^{\omega} \eta^{1-\alpha} G(\eta, s) h(s) d s=\frac{m(t)}{M} \eta^{1-\alpha} u(\eta) .
\end{aligned}
$$

Namely, $t^{1-\alpha} u(t) \geq \frac{m(t)}{M} \eta^{1-\alpha} u(\eta)$.

We define an operator $T: C_{1-\alpha}(I, E) \rightarrow C_{1-\alpha}(I, E)$ as following

$$
T u(t)=\int_{0}^{\omega} G(t, s)(f(s, u(s))+M u(s)) d s=(S \circ F)(u)=S(F(u)) .
$$

Evidently, operator $T: C_{1-\alpha}(I, E) \rightarrow C_{1-\alpha}(I, E)$ is continuous and the solution of problem (1) is equivalent to the non-zero fixed point of operator $T$ defined by (7).

Nextly, we will use the fixed point index theory of condensed mapping to find the fixed point of operator $T$. For this reason, we need to prove that $T$ is condensed mapping. Therefore, we assume $f$ satisfies the following assumption. 
(P0) For any $R>0, f(I \times(\bar{B}(\theta, R)))$ is bounded in $C_{1-\alpha}(I, E)$ and there exist constant $L$, such that for arbitrarily $D \subset K \cap \bar{B}(\theta, R)$ and $t \in I$, we have

$$
\mu_{C_{1-\alpha}}(\{f(t, D)+M D\}) \leq L \mu_{C_{1-\alpha}}(D), \quad t \in I,
$$

where $0<L<\frac{M}{4}, \bar{B}(\theta, R)$ is a closed sphere with $\theta$ as the center, and $R$ as the radius in Banach space $E$.

Lemma 2.10. Assume that the assumption (P0) holds, then $T: C_{1-\alpha}(I, E) \rightarrow C_{1-\alpha}(I, E)$ is a condensed mapping.

Proof. We know that $T$ maps bounded set in $C_{1-\alpha}(I, E)$ into bounded and equicontinuous set in $C_{1-\alpha}(I, E)$, When the assumption (P0) holds. For every non-relatively compact bounded set $B \subset C_{1-\alpha}(I, E)$, we need prove that $\mu_{C_{1-\alpha}}(T(B))<\mu_{C_{1-\alpha}}(B)$. For any bounded set $B \subset C_{1-\alpha}(I, E)$, by the Lemma 2.3, there exists a countable subset $B_{1}=\left\{u_{n}\right\} \subset B$ such that $\mu_{C_{1-\alpha}}(T(B))<\mu_{C_{1-\alpha}}\left(T\left(B_{1}\right)\right)$. Since $T(B)$ is bounded and equicontinuous set, we have

$$
\mu_{C_{1-\alpha}}(B)=\max _{t \in I} \mu\left(t^{1-\alpha} B(t)\right), \quad \mu_{C_{1-\alpha}}(T(B))=\max _{t \in I} \mu\left(t^{1-\alpha} T(B(t))\right) .
$$

For any $t \in I$, we can obtain

$$
\begin{aligned}
\mu\left(t^{1-\alpha} T\left(B_{1}\right)(t)\right) & =\mu\left(\left\{t^{1-\alpha} \int_{0}^{\omega} G(t, s)\left(f\left(s, u_{n}(s)\right)+M u_{n}(s)\right) d s \mid n \in \mathbb{N}\right\}\right) \\
& \leq 2 t^{1-\alpha} \int_{0}^{\omega} G(t, s) \mu\left(f\left(s, B_{1}(s)\right)+M B_{1}(s)\right) d s .
\end{aligned}
$$

By the properties of the measure of noncompactness and condition (P0), we can obtain

$$
\mu_{C_{1-\alpha}}\left(f\left(s, B_{1}(s)\right)+M B_{1}(s)\right) \leq L_{2} \mu_{C_{1-\alpha}}\left(B_{1}(s)\right) \leq L_{2} \mu_{C_{1-\alpha}}\left(B_{1}\right) \leq L_{2} \mu_{C_{1-\alpha}}(B),
$$

and then

$$
\begin{aligned}
\mu\left(t^{1-\alpha} T\left(B_{1}\right)(t)\right) & \leq 2 L_{2} t^{1-\alpha} \int_{0}^{\omega} G(t, s) \mu\left(B_{1}(s)\right) d s \\
& \leq 2 L_{2} t^{1-\alpha} \int_{0}^{\omega} G(t, s) s^{\alpha-1} d s \mu_{C_{1-\alpha}}(B)=\frac{2 L_{2}}{M} \mu_{C_{1-\alpha}}(B) .
\end{aligned}
$$

This above inequality implies

$$
\mu_{C_{1-\alpha}}\left(T\left(B_{1}\right)\right) \leq \frac{2 L_{2}}{M} \mu_{C_{1-\alpha}}(B) .
$$

Since $0<L_{2}<\frac{M}{4}$, so we get

$$
\mu_{C_{1-\alpha}}(T(B)) \leq 2 \mu_{C_{1-\alpha}}\left(T\left(B_{1}\right)\right) \leq \frac{4 L_{2}}{M} \mu_{C_{1-\alpha}}(B)<\mu_{C_{1-\alpha}}(B) .
$$

Hence, $T: C_{1-\alpha}(I, E) \rightarrow C_{1-\alpha}(I, E)$ is a condensing mapping.

Let $P$ be a cone in $C_{1-\alpha}(I, K)$ defined by

$$
P=\left\{u \in C_{1-\alpha}(I, K) \mid t^{1-\alpha} u(t) \geq \frac{m(t)}{M} \eta^{1-\alpha} u(\eta), \quad t, \eta \in I\right\} .
$$


Lemma 2.11. If $f(I \times K) \subset K$, then $T(P) \subset P$.

Proof. For any $t, s \in I$ and $u \in P$, from Lemma 2.9, we have

$$
\begin{aligned}
T u(t) & =\int_{0}^{\omega} G(t, s)(f(s, u(s))+M u(s)) d s \\
& \leq t^{\alpha-1} M \int_{0}^{\omega} e_{\alpha}^{-M(\omega-s)}(f(s, u(s))+M u(s)) d s,
\end{aligned}
$$

therefore

$$
\begin{aligned}
t^{1-\alpha} T u(t) & =\int_{0}^{\omega} t^{1-\alpha} G(t, s)(f(s, u(s))+M u(s)) d s \\
& \geq m(t) \int_{0}^{\omega} e_{\alpha}^{-M(\omega-s)}(f(s, u(s))+M u(s)) d s \geq \frac{m(t)}{M} s^{1-\alpha} T u(s) .
\end{aligned}
$$

Hence, $T(P) \subset P$.

For $0<r<R<\infty$, set

$$
\Omega_{1}=\left\{u \in C_{1-\alpha}(I, E) \mid\|u\|_{C_{1-\alpha}}<r\right\}, \quad \Omega_{2}=\left\{u \in C_{1-\alpha}(I, E) \mid\|u\|_{C_{1-\alpha}}<R\right\},
$$

and

$$
\partial \Omega_{1}=\left\{u \in C_{1-\alpha}(I, E) \mid\|u\|_{C_{1-\alpha}}=r\right\}, \quad \partial \Omega_{2}=\left\{u \in C_{1-\alpha}(I, E) \mid\|u\|_{C_{1-\alpha}}=R\right\} .
$$

Which are the relative boundary of $\Omega_{1}, \Omega_{2}$ in $P$, respectively. Then the fixed point of $T$ in $P \cap\left(\Omega_{2} \backslash \bar{\Omega}_{1}\right)$ is the positive solution of boundary value problem (1). We give following some properties of the fixed point index.

Lemma 2.12. ([26]) Let $\Omega \subset E$ be a bounded open subset of $E$ and let $Q: P \cap \bar{\Omega} \rightarrow P$ be a condensing mapping. If $Q$ satisfies

$$
u \neq \mu Q u, \quad \forall u \in P \cap \partial \Omega, \quad 0<\mu \leq 1
$$

then $i(Q, P \cap \Omega, P)=1$.

Lemma 2.13. ([26]) Let $E$ is Banach space, $P$ is the cone in $E$. Let $Q: P \cap \bar{\Omega} \rightarrow P$ be a condensing mapping. If $Q$ satisfies

$$
Q u \nless u, \quad u \in \partial P \cap \Omega,
$$

then $i(Q, P \cap \Omega, P)=0$.

We will use the fixed point index theory of condensing mapping to find the fixed point of $T$ in $P \cap\left(\Omega_{2} \backslash \bar{\Omega}_{1}\right)$. 


\section{Main results}

Theorem 3.1. Let $E$ be an order Banach space, its positive cone $K$ be a normal cone, and the nonlinear term $f: I \times K \rightarrow K$ is continuous. If (P0) and the following two conditions are satisfied

(P1) There exist constants $0<a<M$ and $\delta>0$ such that $f(t, x) \leq-a x$ for any $t \in I$ and $x \in P \cap\left(\Omega_{2} \backslash \overline{\Omega_{1}}\right)$,

(P2) There exist $b>\frac{M^{2}}{m(t)}-M$ and $H>0$ such that $f(t, x) \geq b x$ for any $t \in I$ and $\|x\|_{C_{1-\alpha}} \geq H$.

Then the boundary value problem (1) has at least one positive solution.

Proof. We just need to prove that the operator $T$ has a fixed point in $P \cap\left(\Omega_{2} \backslash \bar{\Omega}_{1}\right)$ when $r$ is small enough and $R$ large enough.

On the one hand, let $r \in(0, \delta)$, where $\delta$ is the constant in condition (P1). We show that

$$
u-\mu T u \neq \theta, \quad u \in P \cap \partial \Omega_{1}, \quad 0<\mu \leq 1 .
$$

In fact, if there exist $u_{0} \in P \cap \partial \Omega_{1}$ and $0<\mu_{0} \leq 1$ such that $u_{0}=\mu_{0} T u_{0}$, then by the definition of $T$ and the condition (P1), it follows that

$$
\begin{aligned}
u_{0}(t) & =\mu_{0} T u_{0}(t) \leq \int_{0}^{\omega} G(t, s)\left(f\left(s, u_{0}(s)\right)+M u_{0}(s)\right) d s \\
& \leq(M-a) \int_{0}^{\omega} G(t, s) u_{0}(s) d s=(M-a)\left(S u_{0}\right)(t),
\end{aligned}
$$

The above inequalities are used repeatedly, we can obtain

$$
u_{0}(t) \leq(M-a)\left(S u_{0}\right)(t) \leq \cdots \leq(M-a)^{n}\left(S^{n} u_{0}\right)(t), \quad t \in I, \quad n \in \mathbb{N} .
$$

According to the normality of $K$ and Lemma 2.7, we have

$$
\begin{aligned}
\left\|u_{0}\right\|_{C_{1-\alpha}} & \leq N(M-a)^{n}\left\|S^{n}\right\|_{C_{1-\alpha}}\left\|u_{0}\right\|_{C_{1-\alpha}} \leq N(M-a)^{n}\|S\|_{C_{1-\alpha}}^{n}\left\|u_{0}\right\|_{C_{1-\alpha}} \\
& =\left(\frac{M-a}{M}\right)^{n} N\left\|u_{0}\right\|_{C} \rightarrow 0 \quad \text { as } \quad n \rightarrow \infty .
\end{aligned}
$$

The above inequality means that $\left\|u_{0}\right\|_{C_{1-\alpha}} \equiv 0$, which contradicts with the $u_{0} \in P \cap \partial \Omega_{1}$. This mean (8) holds. Therefore, from Lemma 2.12 implies that

$$
i\left(T, P \cap \Omega_{1}, P\right)=1 \text {. }
$$

On the other hand, by the condition (P2), we have

$$
f(t, x) \geq b x, \quad t \in I, \quad\|x\|_{C_{1-\alpha}} \geq H .
$$


We choose $R>\max \left\{\frac{N M H}{m(t)}, r\right\}$, for any $u \in \partial \Omega_{2}$, that is $\|u\|_{C_{1-\alpha}}=R$. For any $s, \tau \in I$, we can obtain

$$
s^{1-\alpha} u(s) \geq \frac{m(t)}{M} \tau^{1-\alpha} u(\tau) .
$$

By the normality of $K$, we have

$$
N\left\|s^{1-\alpha} u(s)\right\| \geq \frac{m(t)}{M}\left\|\tau^{1-\alpha} u(\tau)\right\|, \quad s, \tau \in I .
$$

Take the maximum norm for $\tau$ on both sides of the above formula, it follows that

$$
\left\|s^{1-\alpha} u(s)\right\| \geq \frac{m(t)}{N M}\|u\|_{C_{1-\alpha}} \geq \frac{m(t)}{N M} R \geq H .
$$

From the above formula, (6) and (10), it follows that

$$
\begin{aligned}
t^{1-\alpha} T u(t) & =t^{1-\alpha} \int_{0}^{\omega} G(t, s)(f(s, u(s))+M u(s)) d s \geq(b+M) t^{1-\alpha} \int_{0}^{\omega} G(t, s) u(s) d s \\
& \geq \frac{(b+M) m(t)}{M} t^{1-\alpha} \int_{0}^{\omega} G(t, s) s^{\alpha-1} \tau^{1-\alpha} u(\tau) d s=\frac{(b+M) m(t)}{M^{2}} \tau^{1-\alpha} u(\tau) .
\end{aligned}
$$

Since $b>\frac{M^{2}}{m(t)}-M$, let's take $\tau=t$ in the above formula, we have

$$
t^{1-\alpha} T u(t) \geq t^{1-\alpha} u(t) .
$$

Hence, $T u \nless u$. By Lemma 2.13, we have

$$
i\left(T, P \cap \Omega_{2}, P\right)=0 .
$$

Now, by the additivity of fixed point index, (9) and (11), we can obtain

$$
i\left(T, P \cap\left(\Omega_{2} \backslash \bar{\Omega}_{1}\right), P\right)=i\left(T, P \cap \Omega_{2}, P\right)-i\left(T, P \cap \Omega_{1}, P\right)=-1 .
$$

Consequently, $T$ exists a fixed point in $P \cap\left(\Omega_{2} \backslash \bar{\Omega}_{1}\right)$ which is a positive solution of boundary value problem (1).

Theorem 3.2. Let $E$ be an order Banach space, its positive cone $K$ be a normal cone, and the nonlinear term $f: I \times K \rightarrow K$ is continuous. If (P0) and the following two conditions are satisfied

(P3) There exist constant $b>\frac{M^{2}}{m(t)}-M$ and $\delta>0$ such that $f(t, x) \geq b x$ for any $t \in I$, $x \in K$ and $\|x\|_{C_{1-\alpha}} \geq \delta$.

(P4) There exist constants $0<a<M$ and $h_{1} \in C_{1-\alpha}(I, K)$ such that $f(t, x) \leq-a x+$ $h_{1}(t)$ for any $t \in I$ and $x \in K$.

Then the boundary value problem (1) has at least one positive solution.

Proof. We need to prove that the operator $T$ has a fixed point in $P \cap\left(\Omega_{2} \backslash \bar{\Omega}_{1}\right)$ when $r$ is small enough and $R$ large enough. 
On the one hand, we choose $0<r<\min \left\{\frac{N M H}{m(t)}, r\right\}$. For any $u \in \partial \Omega_{1}$ and $s, \tau \in I$, by the condition (P3) and (6), we have

$$
\begin{aligned}
t^{1-\alpha} T u(t) & =t^{1-\alpha} \int_{0}^{\omega} G(t, s)(f(s, u(s))+M u(s)) d s \geq(b+M) t^{1-\alpha} \int_{0}^{\omega} G(t, s) u(s) d s \\
& \geq \frac{(b+M) m(t)}{M} t^{1-\alpha} \int_{0}^{\omega} G(t, s) s^{\alpha-1} \tau^{1-\alpha} u(\tau) d s=\frac{(b+M) m(t)}{M^{2}} \tau^{1-\alpha} u(\tau) .
\end{aligned}
$$

Owing to $b>\frac{M^{2}}{m(t)}-M$, we take $\tau=t$ in the above inequality, we have

$$
T u(t)>u(t) .
$$

Hence, $T u(t) \nless u(t)$. By Lemma 2.13, we can obtain

$$
i\left(T, P \cap \Omega_{1}, P\right)=0 .
$$

On the other hand, we show that following formula holds when $R$ is large enough.

$$
u-\mu T u \neq \theta, \quad u \in P \cap \partial \Omega_{2}, \quad 0<\mu \leq 1 .
$$

Actually, if (13) dose not hold, there exist $u_{1} \in P \cap \partial \Omega_{2}$ and $0<\mu_{1} \leq 1$ such that $u_{1}=\mu_{1} T u_{1}$. For any $t \in I$, from the definition of $T$ and the condition (P4), we have

$$
\begin{aligned}
u_{1}(t) & =\mu_{1} T u_{1}(t) \leq \int_{0}^{\omega} G(t, s)\left(f\left(s, u_{1}(s)\right)+M u_{1}(s)\right) d s \\
& \leq \int_{0}^{\omega} G(t, s)\left[(M-a) u_{1}(s)+h_{1}(s)\right] d s=(M-a)\left(S u_{1}\right)(t)+\int_{0}^{\omega} G(t, s) h_{1}(s) d s,
\end{aligned}
$$

therefore

$$
(I-(M-a) S) u_{1}(t) \leq \int_{0}^{\omega} G(t, s) h_{1}(s) d s=S h_{1}(t)
$$

Because of $\|S\|_{C_{1-\alpha}} \leq \frac{1}{M}$, then $\|(M-a) S\|_{C_{1-\alpha}} \leq 1$. According to the perturbation theorem, $I-(M-a) S$ has a bounded inverse operator $(I-(M-a) S)^{-1}$, and

$$
(I-(M-a) S)^{-1}=\sum_{n=0}^{\infty}[(M-a) S]^{n}
$$

is a positive operator. Acting on $(14)$ by $(I-(M-a) S)^{-1}$, we have

$$
\theta \leq u_{1}(t) \leq(I-(M-a) S)^{-1}\left(S h_{1}\right)(t) .
$$

And since the normality of cone $K$, we have

$$
\begin{aligned}
\left\|t^{1-\alpha} u_{1}(t)\right\| & \leq N\left\|(I-(M-a) S)^{-1} t^{1-\alpha}\left(S h_{1}\right)(t)\right\| \leq N\left\|(I-(M-a) S)^{-1}\left(S h_{1}\right)\right\|_{C_{1-\alpha}} \\
& \leq N\left\|(I-(M-a) S)^{-1}\right\|_{C_{1-\alpha}}\left\|S h_{1}\right\|_{C_{1-\alpha}} \\
& \leq N \sum_{n=0}^{\infty}\left\|(M-a)^{n} S^{n}\right\|_{C_{1-\alpha}}\left\|S h_{1}\right\|_{C_{1-\alpha}},
\end{aligned}
$$


therefore

$$
\left\|u_{1}\right\|_{C_{1-\alpha}} \leq \frac{N\left\|h_{1}\right\|_{C_{1-\alpha}}}{M} \sum_{n=0}^{\infty}\left\|(M-a)^{n} S^{n}\right\|_{C_{1-\alpha}} .
$$

There exist $0<a<M$ such that $M-a<M$, it implies that

$$
\left\|(M-a)^{n} S^{n}\right\|_{C_{1-\alpha}} \leq\left(\frac{M-a}{M}\right)^{n} .
$$

Obviously, $\sum_{n=0}^{\infty}\left(\frac{M-a}{M}\right)^{n}$ is convergent, that is, $\sum_{n=0}^{\infty}(M-a)^{n}\left\|S^{n}\right\|_{C_{1-\alpha}}$ is convergent. Denote $M_{0}=\sum_{n=0}^{\infty}\left\|(M-a)^{n} S^{n}\right\|_{C_{1-\alpha}}<+\infty$, it follows that

$$
\left\|u_{1}\right\|_{C_{1-\alpha}} \leq \frac{N\left\|h_{1}\right\|_{C_{1-\alpha}}}{M} M_{0}
$$

We choose $R>\max \left\{\delta, \frac{N\left\|h_{1}\right\|_{C_{1-\alpha}}}{M} M_{0}\right\}$, then (14) holds. From Lemma 2.12, we have

$$
i\left(T, P \cap \Omega_{2}, P\right)=1 \text {. }
$$

By the additivity of fixed point index, (12) and (15), we can obtain

$$
i\left(T, P \cap\left(\Omega_{2} \backslash \bar{\Omega}_{1}\right), P\right)=i\left(T, P \cap \Omega_{2}, P\right)-i\left(T, P \cap \Omega_{1}, P\right)=1 .
$$

Therefore, $T$ exists a fixed point in $P \cap\left(\Omega_{2} \backslash \bar{\Omega}_{1}\right)$, which is a positive solution of boundary value problem (1).

Acknowledgements. The authors would like to express sincere thanks to the anonymous referee for his/her carefully reading the manuscript and valuable comments and suggestions. This work is supported by Natural Science Foundation of Gansu Province (No. 20JR5RA522), Science Research Project for Colleges and Universities of Gansu Province (No. 2019B-047), Project of NWNU-LKQN2019-13 and Doctoral Research Fund of Northwest Normal University (No. 6014/0002020209).

\section{REFERENCES}

[1] R.L. Magin, Fractional calculus in bioengineering, Crit. Rev. Biomed. Eng., 32:1-104, 2004.

[2] B.M. Vinagre, I. Podlubny, A. Hernández and V. Feliu, Some approximations of fractional order operators used in control theory and applications, Fract. Calc. Appl. Anal., 3:231-248, 2000.

[3] K.B. Oldham, Fractional differential equations in electrochemistry, Adv. Eng. Softw., 41:9-12, 2010.

[4] R. Metzler and J. Klafter, Boundary value problems for fractional diffusion equations, Phys. A., 278:107-125, 2000.

[5] X. Hao, Positive solution for singular fractional differential equations involving derivatives, Adv. Differ. Equ., 139:1-12, 2016.

[6] H. Afshari, H. Marasi and H. Aydi. Existence and uniqueness of positive solutions for boundary value problems of fractional differential equations, Filomat, 31:2675-2682, 2017. 
[7] A.A. Kilbas, H.M. Srivastava and J.J. Trujillo, Theory and applications of fractinal differential equations, Elsevier Science B. V., Amsterdam, 2006.

[8] X. Hao and H. Wang, Positive solutions of semipositone singular fractional differential systems with a parameter and integral boundary conditions, Open Math., 16:581-596, 2018.

[9] P. Chen, X. Zhang and Y. Li, Study on fractional non-autonomous evolution equations with delay, Comput. Math. Appl., 73:794-803, 2017

[10] P. Chen, X. Zhang and Y. Li, A blowup alternative result for fractional nonautonomous evolution equation of Volterra type, Commun. Pure Appl. Anal., 17:1975- 1992, 2018.

[11] P. Chen, X. Zhang and Y. Li, Fractional non-autonomous evolution equation with nonlocal conditions, J. Pseudo-Differ.Oper. Appl., 10: 955-973, 2019.

[12] P. Chen, X. Zhang and Y. Li, Approximate controllability of non-autonomous evolution system with nonlocal conditions, J. Dyn. Control. Syst., 26:1-16, 2020.

[13] P. Chen, X. Zhang and Y. Li, Existence and approximate controllability of fractional evolution equations with nonlocal conditions via resolvent operators, Fract. Calcu. Appl. Anal., 23:268-291, 2020.

[14] P. Chen, X. Zhang and Y. Li, Cauchy problem for fractional non-autonomous evolution equations, Banach J. Math. Anal., 14:559-584, 2020.

[15] Z. Bai and H. Lü, Positive solutions for boundary value problem of nonlinear fractional differential equation, J. Math. Anal. Appl., 311:495-505, 2005.

[16] A. Cabada and Z. Hamdi, Existence results for nonlinear fractional Dirichlet problems on the right side of the first eigenvalue, Georgian Math. J., 24:41-53, 2017.

[17] Y. Li and C. Guo, Positive solutions for nonlinear second order boundary value problems in oedered Banach spaces, J. Lanzhou University(Natural Sciences), 44:120-123, 2008.

[18] D. Guo and V. Lakshmikantham, Multiple solutions of two-point boundary value problems of ordinary differential equations in Banach spaces, J. Math. Anal. Appl., 129:211-222, 1988.

[19] J. Banas̀ and K. Goebel, Measures of Noncompactness in Banach Spaces, In Lecture Notes in Pure and Applied Mathematics, Volume 60, Marcel Dekker, New York, 1980.

[20] K. Deimling, Nonlinear Functional Analysis, Springer-Verlag, New York, 1985.

[21] I. Podlubny, Fractional differential equations, Academic Press, San Diego, 1999.

[22] A. Cabada and T. Kisela, Existence of positive periodic solutions of some nonlinear fractional differential equations, Commun. Nonlinear Sci. Numer. Simul., 50:51-67, 2017.

[23] H.P. Heinz, On the behaviour of measure of noncompactness with respect to differentiation and integration of vector-valued functions, Nonlinear Anal., 7:1351-1371, 1983.

[24] Y. Li, Existence of solutions of initial value problem for abstract semilinear evolution equations, Acta. Nath. Sin., 48:1089-1094, 2005.

[25] P. Chen and Y. Li, Monotone iterative thchnique for a class of semilinear evolution equations with nonlocal conditions, Results Math., 63:731-744, 2013.

[26] D. Guo and V. Lakshmikantham, Nonlinear Problem in Abstract Cones, Academic Press, New York, 1988. 